\title{
ACOUSTIC POINT-SOURCE REFLECTION FROM A SEABED WITH A NON- UNIFORM FLUID-LIKE SEDIMENT LAYER
}

Jin-Yuan Liu

Institute of Undersea Technology, National Sun Yat-sen University, and NCKU-NSYSU Research Center for Ocean Environment and Technology, 70 Lian-Hae Road, Kaohsiung, Taiwan 804, R.O.C., jimliu@mail.nsysu.edu.tw

Chung-Ray Chu

Institute of Undersea Technology, National Sun Yat-sen University, and NCKU-NSYSU Research Center for Ocean Environment and Technology, 70 Lian-Hae Road, Kaohsiung, Taiwan 804, R.O.C.

Yung-Hong Wu

Institute of Undersea Technology, National Sun Yat-sen University, and NCKU-NSYSU Research Center for Ocean Environment and Technology, 70 Lian-Hae Road, Kaohsiung, Taiwan 804, R.O.C.

Hsin-Hung Chen

Institute of Undersea Technology, National Sun Yat-sen University, and NCKU-NSYSU Research Center for Ocean Environment and Technology, 70 Lian-Hae Road, Kaohsiung, Taiwan 804, R.O.C.

Follow this and additional works at: https://jmstt.ntou.edu.tw/journal

Part of the Engineering Commons

\section{Recommended Citation}

Liu, Jin-Yuan; Chu, Chung-Ray; Wu, Yung-Hong; and Chen, Hsin-Hung (2005) "ACOUSTIC POINT-SOURCE REFLECTION FROM A SEABED WITH A NON-UNIFORM FLUID-LIKE SEDIMENT LAYER," Journal of Marine Science and Technology. Vol. 13: Iss. 3, Article 8.

DOI: $10.51400 / 2709-6998.2129$

Available at: https://jmstt.ntou.edu.tw/journal/vol13/iss3/8

This Research Article is brought to you for free and open access by Journal of Marine Science and Technology. It has been accepted for inclusion in Journal of Marine Science and Technology by an authorized editor of Journal of Marine Science and Technology. 


\section{ACOUSTIC POINT-SOURCE REFLECTION FROM A SEABED WITH A NON- UNIFORM FLUID-LIKE SEDIMENT LAYER}

\section{Acknowledgements}

This work was supported in part by National Science Council of Taiwan, R.O.C. through contract NSC91-2611-U110-005 and the Joint Project of National Cheng-Kung University and National Sun Yatsen University sponsored by the Ministry of Education of Taiwan, R.O.C. The authors would like to express our profound thanks for their financial supports. 


\title{
ACOUSTIC POINT-SOURCE REFLECTION FROM A SEABED WITH A NON-UNIFORM FLUID-LIKE SEDIMENT LAYER
}

\author{
Jin-Yuan Liu*, Chung-Ray Chu*, Yung-Hong Wu*, and Hsin-Hung Chen*
}

\begin{abstract}
Key words: point-source reflection, non-uniform sediment layer, $k^{2}$-linear sound speed profile, inverse-square sound speed profile, elastic basement, interface wave.
\end{abstract}

\section{ABSTRACT}

Acoustic point-source reflection from a non-uniform seabed within which the density and sound speed vary continuously with depth is considered in this paper. This fundamental problem has illuminated many interesting phenomena about the effects of sediment stratification on acoustic wave interactions with the seabed. The analysis employs the analytical solutions for the Helmholtz equation in the sediment layer, in which the density and the sound speed are, respectively, described by three kinds of geological models. The results have demonstrated that the effects of sediment stratification are confined in a region in range, where the reflection field is dominated by the reflected ray bundles containing ray components with the grazing angles that are neither too steep nor too shallow. The effects of many other factors, such as frequency, sediment thickness, basement elasticity, on the reflection field are investigated; in particular, the interface wave component may become important in the region close to the seabed, if the sediment thickness is relatively thin in comparison with the acoustic wave length.

\section{INTRODUCTION}

The geoacoustic properties of seabed, including geological and geometrical characteristics, affect significantly the acoustic wave propagation in an ocean waveguide, particularly in a shallow water environment. The interactions of acoustic wave with seabed, which in general are non-uniform and/or randomly inhomogeneous, inevitably result in a reduction of acoustic energy as well as sound field coherency. As a result, the subject of acoustic wave interactions with non-uniform seabed has constantly attracted the attention in the study of ocean acoustics. The present analysis consid-

Paper Submitted 09/09/04, Accepted 04/29/05. Author for Correspondence: Jin-Yuan Liu. E-mail: jimliu@mail.nsysu.edu.tw.

*Institute of Undersea Technology, National Sun Yat-sen University, and NCKU-NSYSU Research Center for Ocean Environment and Technology, 70 Lian-Hae Road, Kaohsiung, Taiwan 804, R.O.C. ers a fundamental problem of acoustic point-source reflection from a seabed with non-uniform distributions of density and sound speed; the environmental geometry is schematically shown in Figure 1 . The sediment layer is assumed to be fluid-like, within which the acoustic properties may be portrayed by a parameterized model capable of describing a realistic seafloor [3], and at the same time, rendering analytical solutions to the acoustic wave equation. While the solutions completely based upon numerical computation are readily obtained for a general horizontallystratified oceanic environment, the parameterized modeling offers a better approach for the seabed-property analysis, particularly in the applications of geoacoustic inversion.

The problem of acoustic plane wave incident upon a seabed, such as the one shown in Figure 1, has been analyzed in a sequence of papers by Robins [7-10], and later extended by Liu and Huang [5], Liu et al. [6], to include random roughness on the surface and/or shear elasticity within the seabed. These studies have developed the analytical expressions corresponding to various density and sound speed profiles, and implemented the formulations incorporating with the current software to analyze the reflection and/or scattering sound

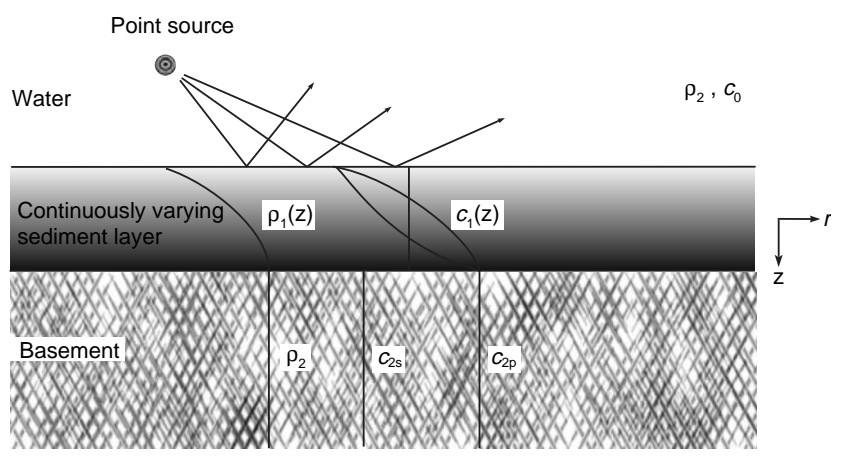

Fig. 1. Environmental model: a point-source wave incident upon a seabed with a fluid-like sediment layer, subject to continuous variations in density and sound speed. 
fields. The present analysis is a direct extension of the aforementioned studies for the source geometry from the plane wave to the point source. Although the extension is rather straightforward, the point source sound field covering full wavenumber spectrum, in stark contrast to the single-wavenumber-component plane wave, is expected to demonstrate a more complicated interfering pattern in the reflection field, which is the primary objective in this analysis.

In the following sections, the formulations to be employed in this analysis are first summarized. Then the computational algorithms are implemented to generate numerical results, which are gauged by current available programs to ensure their correctness. Numerical examples are demonstrated and discussed.

\section{FORMULATIONS}

Consider an acoustic point source with a spectral strength $S_{\omega}$ in a uniform medium with density $\rho_{0}$ and sound speed $c_{0}$, located at a distance $z_{s}$ above the seabed. The seabed consists of a fluid-like sediment layer of thickness $h$, and with the density $\rho_{1}(z)$ and the sound speed $c_{1}(z)$ both varying continuously with depth in a specific fashion to be described later. The seabed overlies on a semi-infinite basement which is assumed to be a uniform elastic medium with density $\rho_{2}$, compressional sound speed $c_{2 p}$, and shear sound speed $c_{2 s}$. The coordinate system takes $z$-axis passing through the source, so that the overall sound field is axisymmetric with respect to $z$-axis; the overall environmental model is shown in Figure 1.

\section{Solutions of acoustic wave equations}

By the principle of Fourier decomposition, the time-dependent acoustic sound field in each layer may be represented as:

$$
\phi_{i}(r, z, t)=\int \phi_{i, \omega}(r, z) e^{i \omega t} d \omega, \quad i=0,1,2
$$

where $\phi_{i}$ represents an acoustic variable in layer $i$, which for convenience is taken to be the acoustic pressure in a fluid layer and the displacement potential in an elastic layer, and $\phi_{i, \omega}$ is the corresponding frequency spectrum satisfying the Helmholtz equation in various layers [2]:

$$
\begin{aligned}
& \left(\nabla^{2}+k_{0}^{2}\right) \phi_{0, \omega}=S_{\omega} \frac{\delta(r) \delta\left(z-z_{s}\right)}{2 \pi r} \\
& \left(\nabla^{2}-\left(\frac{1}{\rho} \frac{d \rho_{1}}{d z}\right) \frac{\partial}{\partial z}+k_{1}^{2}(z)\right) \phi_{1, \omega}=0
\end{aligned}
$$

$$
\begin{aligned}
& \left(\nabla^{2}+k_{2 p}^{2}\right) \phi_{2 p, \omega}=0 \\
& \left(\nabla^{2}+k_{2 s}^{2}\right) \phi_{2 s, \omega}=0
\end{aligned}
$$

where $k_{o}=\omega / c_{0}$ is the medium wavenumber of the upper fluid layer, and $k_{1}(z)=\omega / c_{1}(z)$ is that of the fluid-like sediment layer, which is a function of $z$, and $k_{2 p}=\omega / c_{2 p}$ and $k_{2 s}=\omega / c_{2 s}$ are, respectively, the compressional and shear wavenumbers is the lower elastic layer. It is noted that the second term in the Eq. (3) represents the effects of density variation in the medium.

In view of range-independent characteristics of the environment as well as axisymmetic nature of the sound field, the solutions of the Helmholtz equations may be expressed as Fourier-Bessel representation [4]:

$$
\phi_{i, \omega}=\int_{0}^{\infty} \tilde{\phi}_{i, \omega}\left(k_{r}, z\right) J_{0}\left(k_{r} r\right) k_{r} d k_{r}
$$

where $\tilde{\phi}_{i, \omega}$ is the $i$ th-layer wavenumber spectrum for frequency $\omega, J_{0}$ is the zeroth-order Bessel function of the first kind, and $k_{r}$ is the horizontal wavenumber. Substituting Eq. (6) into the corresponding Helmholtz equations, Eqs. (2)-(5), results in the following depthdependent equations:

$$
\begin{aligned}
& \left(\frac{d^{2}}{d z^{2}}+k_{0, z}^{2}\right) \tilde{\phi}_{0, \omega}=S_{\omega} \frac{\delta\left(z-z_{s}\right)}{2 \pi} \\
& \left(\frac{d^{2}}{d z^{2}}+\left(\frac{1}{\rho_{1}} \frac{d \rho_{1}}{d z}\right) \frac{d}{d z}+k_{1, z}^{2}(z)\right) \tilde{\phi}_{1, \omega}=0 \\
& \left(\frac{d^{2}}{d z^{2}}+k_{2 p, z}^{2}\right) \tilde{\phi}_{2 p, \omega}=0 \\
& \left(\frac{d^{2}}{d z^{2}}+k_{2 s, z}^{2}\right) \tilde{\phi}_{2 s, \omega}=0
\end{aligned}
$$

where $k_{1, z}=\left(k_{i}^{2}-k_{r}^{2}\right)^{1 / 2}$ is the vertical wavenumber in the layer $i$.

The exact solutions for the depth-dependent equations are readily derived for layers 0 and 2 , since the corresponding vertical wavenumbers are constant; however, for layer 1, the solution may only be obtained through a numerical procedure for general variations of density and/or sound speed with respect to depth. Based upon the fundamental theorem for the linear ordinary differential equations [1], the general solution of Eq. (8) may be expressed as a linear combination of two fundamental solutions, $\mathcal{G}\left(k_{r}, z\right)$ and $\mathcal{H}\left(k_{r}, z\right)$. Therefore, the solutions corresponding to Eqs. (7)-(10) 
are, respectively, given by:

$$
\begin{aligned}
& \tilde{\phi}_{0, \omega}\left(k_{r}, z\right)=A_{0}^{+}\left(k_{r}\right) e^{\alpha_{0} z}-\frac{S_{\omega}}{4 \pi \alpha_{0}} e^{-\alpha_{0}\left|z-z_{S}\right|} \\
& \tilde{\phi}_{1, \omega}\left(k_{r}, z\right)=A_{1}^{-}\left(k_{r}\right) \mathcal{G}\left(k_{r}, z\right)+A_{1}^{+}\left(k_{r}\right) \mathcal{H}\left(k_{r}, z\right) \\
& \tilde{\phi}_{2 p, \omega}\left(k_{r}, z\right)=A_{2}^{-}\left(k_{r}\right) e^{-\alpha_{2 p}(z-h)} \\
& \tilde{\phi}_{2 s, \omega}\left(k_{r}, z\right)=k_{r}^{-1} B_{2}^{-}\left(k_{r}\right) e^{-\alpha_{2 s}(z-h)}
\end{aligned}
$$

where the coefficients in the exponential functions are defined as:

$$
\begin{aligned}
& \alpha_{0}=\left(k_{r}^{2}-k_{0}^{2}\right)^{1 / 2} \\
& \alpha_{2 p}=\left(k_{r}^{2}-k_{2 p}^{2}\right)^{1 / 2} \\
& \alpha_{2 s}=\left(k_{r}^{2}-k_{2 s}^{2}\right)^{1 / 2}
\end{aligned}
$$

The unknown amplitudes $A_{0}^{+}, A_{1}^{-}, A_{1}^{+}, A_{2}^{+}, B_{2}^{-}$may be determined from the boundary conditions, which in present case are:

$$
\begin{aligned}
& \left.w_{0}\right|_{z=0}=\left.w_{1}\right|_{z=0} \\
& \left.p_{0}\right|_{z=0}=\left.p_{1}\right|_{z=0} \\
& \left.w_{1}\right|_{z=h}=\left.w_{2}\right|_{z=h} \\
& \left.p_{1}\right|_{z=h}=-\left.\sigma_{z z}\right|_{z=h} \\
& 0=\left.\sigma_{r z}\right|_{z}=h
\end{aligned}
$$

where $w_{i}, p_{i}$, and $\sigma$ 's represent, respectively, the vertical displacement, acoustic pressure, and stresses in various layers. By the applications of the boundary and the radiation conditions, the linear system for the unknown amplitudes is formed, which may be conveniently cast as:

$$
\tilde{B}\left(k_{r}\right) \tilde{\chi}\left(k_{r}\right)=\tilde{C}\left(k_{r}\right)
$$

where $\tilde{\chi}\left(k_{r}\right)$ is a vector containing the unknowns:

$$
\tilde{\chi}\left(k_{r}\right)=\left[\begin{array}{lllll}
A_{0}^{+}\left(k_{r}\right) & A_{1}^{-}\left(k_{r}\right) & A_{1}^{+}\left(k_{r}\right) & A_{2}^{-}\left(k_{r}\right) & B_{2}^{-}\left(k_{r}\right)
\end{array}\right]^{T}
$$

and the coefficient matrix $\tilde{B}\left(k_{r}\right)$ and the forcing vector $\tilde{C}\left(k_{r}\right)$ are, respectively, given as follows:

$\tilde{B}\left(k_{r}\right)=$

$$
\begin{array}{ccc}
\frac{\alpha_{0}}{\rho_{0} \omega^{2}} & -\frac{1}{\rho_{1 U} \omega^{2}} \mathcal{G}^{\prime}\left(k_{r}, 0\right) & -\frac{1}{\rho_{1 U} \omega^{2}} \mathcal{H}^{\prime}\left(k_{r}, 0\right) \\
1 & -\mathcal{G}\left(k_{r}, 0\right) & -\mathcal{H}\left(k_{r}, 0\right) \\
0 & \frac{1}{\rho_{1 L} \omega^{2}} \mathcal{G}^{\prime}\left(k_{r}, h\right) & \frac{1}{\rho_{1 L} \omega^{2}} \mathcal{H}^{\prime}\left(k_{r}, h\right) \\
0 & \mathcal{G}\left(k_{r}, h\right) & \mathcal{H}\left(k_{r}, h\right) \\
0 & 0 & 0
\end{array}
$$

$$
\begin{array}{cc}
\mu_{2}\left(2 k_{r}^{2}-k_{2 s}^{2}\right) & -2 \mu_{2} k_{r} \alpha_{2 s} \\
2 \mu_{2} k_{r} \alpha_{2 p} & -\mu_{2}\left(2 k_{r}^{2}-k_{2 s}^{2}\right)
\end{array}
$$$$
\tilde{C}\left(k_{r}\right)=\left[-\frac{S_{\omega}}{4 \pi \rho_{0} \omega^{2}} \operatorname{sign}\left(-z_{s}\right) e^{-\alpha_{0}|z s|} \frac{S_{\omega}}{4 \pi \alpha_{0}} e^{-\alpha_{0}|z s|} \quad 0 \quad 0 \quad 0\right]^{T}
$$

where $\rho_{1 U}$ and $\rho_{1 L}$ are, respectively, the density at the upper boundary and the lower boundary of the sediment layer, and $\mu_{2}$ is the shear modulus of the basement layer; it is noted that $\rho_{1 L}=\rho_{2}$ to maintain density continuity at the lower boundary. The symbol $\mathcal{G}^{\prime}$ and $\mathcal{H}^{\prime}$ denote the derivative with respect to its argument of the function, and sign( ) represents positive or negative depending on the sign of the numerics inside the argument.

\section{Exact solutions of the depth-denpedent equation in a continuously varying sediment layer}

To proceed the analysis, the functions $\mathcal{G}$ and $\mathcal{H}$, along with their derivatives, in the matrix $\tilde{B}$ in Eq. (22) must be defined. These two functions, as noted in a previous paragraph, are the fundamental solutions of the depth-dependent equation, Eq. (8), in the sediment layer, which in general may only be obtained numerically for an arbitrary variation of density $\rho_{1}(z)$ and/or sound speed $c_{1}(z)$ (equivalently $k_{1}(z)$ ). Here, we are 
interested in the analytical solutions corresponding to various combinations of the density and sound speed profiles which are geologically meaningful. Under this consideration, it has been shown that the exact solutions of Eq. (8) exist if the density and the sound speed profiles satisfy the following combinations [7, $11,12,13]$ :

$$
\begin{aligned}
& \rho_{1}(z)=A \frac{e^{-\alpha(z-h / 2)}}{\left[e^{-\alpha(z-h / 2)}+a\right]^{2}} \\
& \frac{1}{c^{2}(z)}= \begin{cases}\frac{1}{\bar{c}_{1}^{2}}, & \text { constant } \\
\frac{(1+\delta z)}{c_{1 U}^{2}}, & k^{2}-\text { linear } \\
\frac{b^{2}}{c_{0}^{2}}+\left(\frac{1}{c_{1 U}^{2}}-\frac{b^{2}}{c_{0}^{2}}\right) \frac{1}{(1-\kappa z)^{2}}, & \text { inverse - square }\end{cases}
\end{aligned}
$$

where $A, \alpha, a, \bar{c}_{1}, c_{1 U}, \delta, b, c_{0}$, and $\kappa$ are constants, which when appropriately assigned may well fit the profiles in a realistic sea floor [3]. An example of the profiles is shown in Figure 2. It is noted that $c_{1 U}$ and $c_{1 L}$ are the sound speeds at the upper and lower boundary of the sediment layer, respectively. Again, to maintain continuity, $c_{1 U}=c_{2 p}$.

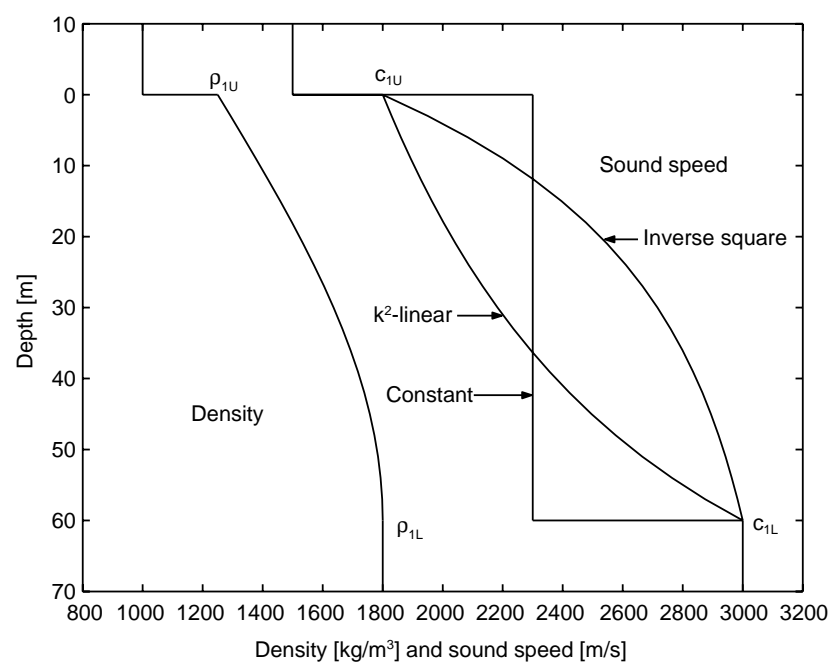

Fig. 2. Sample profiles for density and sound speed; parameters employed (units omitted): $A=2073, \alpha=-0.0249, a=0.288, c_{1}=$ 2059, 1750, 1750 (for constant, $k^{2}$-linear, inverse-square sound speed profile, respectively), $\delta=-0.0101, b=0.953, c_{0}=2500$, and $\kappa=-\mathbf{0 . 0 5}$.
For a plane wave having the horizontal wavenumber $k_{r}=k_{0} \cos \theta_{0}$ with $\theta_{0}$ incident grazing angle, the solution of Eq. (8) may be expressed as:

$$
p(r, z)=\tilde{p}(z) e^{i k_{r} r}
$$

The solutions for $\tilde{p}(z)$ i.e., the functions $\mathcal{G}$ and $\mathcal{H}$ in Eq. (12), corresponding to various profiles shown above have been derived in literature, and may be summarized as follows [7]:

$\tilde{p}_{1}(z)=\sqrt{\rho(z)} \begin{cases}e^{\sigma z}, e^{-\sigma z} & \text { constant } \\ A i(\eta(z)), B i(\eta(z)), & k^{2}-\text { linear } \\ \sqrt{\zeta(z)} H_{v}^{(1)}(\beta \zeta(z)), \sqrt{\zeta(z)} H_{v}^{(2)}(\beta \zeta(z)), & \text { inverse-squa }\end{cases}$

where $A i, B i$ are the Airy functions, and $H_{v}^{(1)}, H_{v}^{(2)}$ are the $v$ th-order Hankel functions; relevant parameters/variables are defined below:

$$
\begin{aligned}
& \sigma^{2}=\frac{\alpha^{2}}{4}-\breve{k}_{r}^{2} \sin ^{2} \theta_{1} \\
& \eta=-\frac{1}{\sqrt[3]{\left(k_{1 U}^{2} \delta\right)^{2}}}\left(-k_{1 U}^{2} \delta z+k_{1 U}^{2}-k_{r}^{2}-\frac{\alpha^{2}}{4}\right) \\
& \zeta=1-\kappa z \\
& \beta^{2}=\frac{1}{\kappa^{2}}\left(k_{0}^{2} b^{2}-k_{r}^{2}-\frac{\alpha^{2}}{4}\right) \\
& v^{2}=\frac{1}{4}-\frac{\omega^{2}}{\kappa^{2}}\left(\frac{1}{c_{1 U}^{2}}-\frac{b^{2}}{c_{0}^{2}}\right)
\end{aligned}
$$

with $k_{1}=\omega / c_{1}$ and $\theta_{1}$ defined as $k_{1} \cos \theta_{1}=k_{0} \cos \theta_{0}$. It is to be noticed from Eq. (32) that for high frequency, or any other factor rendering $v^{2}$ negative, the Hankel function becomes imaginary orders. While both the Airy functions and the Hankel functions are generally builtin in most computational software, the imaginary order of Hankel functions may not be readily available, so that a special treatment may be needed [7]. For this instance, the authors have found that Mathematica [14] is particularly useful in this analysis.

\section{RESULTS AND DISCUSSION}

The formulations derived in the previous section may be readily implemented to investigate the effects of 
sediment stratification on the sound fields in each layer, particularly in the upper semi-infinite half space, which shall be referred to as the reflection field that comprises both the source and reflected fields. In the present case of point source, in order to view the overall behavior of the sound fields, the results are better represented by the Transmission Loss (TL) over the range, as opposed to the reflection coefficient for the case of plane wave. The transmission loss in the upper layer is defined as (Since the absolute value of TL bears little significance in this analysis as the source spectrum $S_{\omega}$ is nominally taken to be unity, so that the unit of TL may considered to be $\mathrm{dB}$ referenced to $1 \mathrm{~Pa}$.):

$$
\mathrm{TL}=20 \log \left|p_{0}\right|
$$

It is to be noted that the source spectrum $S_{\omega}$ shown in Eq. (2) is taken to be unity, which may be considered as the sound field being normalized by the source spectrum.

Before generating the numerical results, when possible, the computational algorithm should be checked against the currently available software to ensure its correctness. In this regard, the widely-applied numerical program OASES is invoked. It is noted that OASES employs a numerical device that discretizes the nonhomogeneous medium into a few numbers of layers, each of which being uniform, and then solves the problem by the method of global matrix. Therefore, by treating the sediment layer as a whole with the exact expressions for the wave equation, the results should be asymptotically approached by those generated by OASES as the grid for the non-homogeneous layer is gradually refined. Figure 3 shows a comparison between the present results, referred to as exact solutions, and those obtained by OASES for a typical example of inversesquare case shown in Figure 2. It is clearly seen in Figure 3 that, as the number of layering increases from 5 to 20 in OASES, the results become closer and closer to the exact solutions, indicating that the numerical algorithm is reliable in the present analysis.

Figure 4 shows the results of TL for frequency 50 $\mathrm{Hz}$, and for three kinds of sound speed stratification; the density distribution remains the same as generalizedexponential type. It is interesting to note that variation in sediment stratification affects the results mainly in the intermediate range, which for the set of parameters shown in the legend of the figure is roughly between 100 and $800 \mathrm{~m}$. This behavior is analyzed as follows. In the field close to the source (near field), the reflection field is dominated by the direct paths from the source field as well as the reflective waves resulting from the steeper source sound rays, for which the reflection is weak and most energy is penetrated into the bottom, and therefore it makes little difference for which type of sound speed variation the sound rays are encountered in the sediment layer. On the other hand, for the sound field far away from the source (far field), the reflection field is in effect attributable to those source sound rays emerating with shallow grazing angles, for which they exercise total reflection without feeling the difference of the sediment stratification. For the intermediate range, the reflection field, besides the direct source field, is an integration of a bundle of reflective (and/or penetrable) sound rays whose amplitudes and/or phases are significantly affected by the sediment properties, rendering the stratification of the sediment layer an effective factor for the characteristics of the reflection field.

Figures 5 and 6 are the similar results as Figure 4

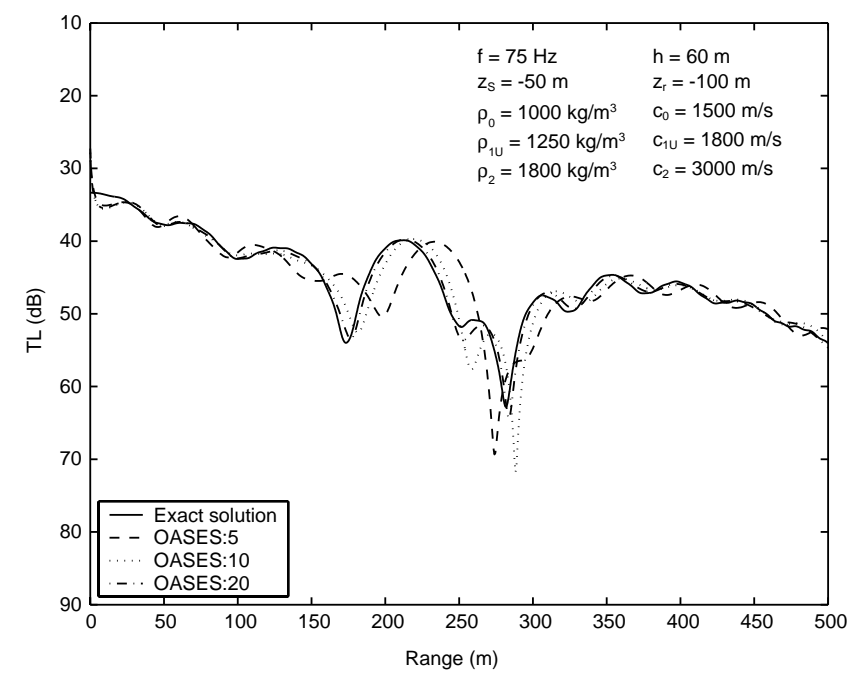

Fig. 3. A comparison between the present results (exact solutions) and those generated by OASES.

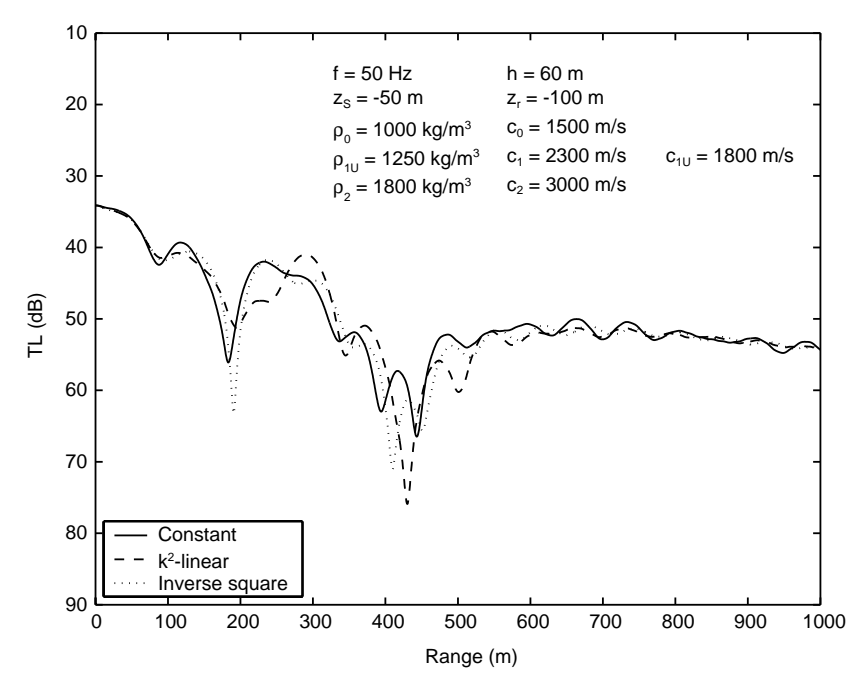

Fig. 4. TL for various sound speed distributions for frequency $50 \mathrm{~Hz}$. 
except for frequency changed to $75 \mathrm{~Hz}$ and $100 \mathrm{~Hz}$, respectively. These results demonstrate similar behaviors as those described in the previous paragraph, except now that the outer bound of the effective range of influence due to sediment stratification is pushed out further as the frequency increases, which is seen, as an example, in Figure 7 for the case of inverse-square profile. It is also noticed that the interference pattern becomes more and more complicated as the frequency increases.

To examine the dependence of reflection field on other geometrical or dynamical parameters, in what follows, we shall just focus on the case of inversesquare sound speed profile in the sediment layer because of its bearing close to reality. Figure 8 shows the

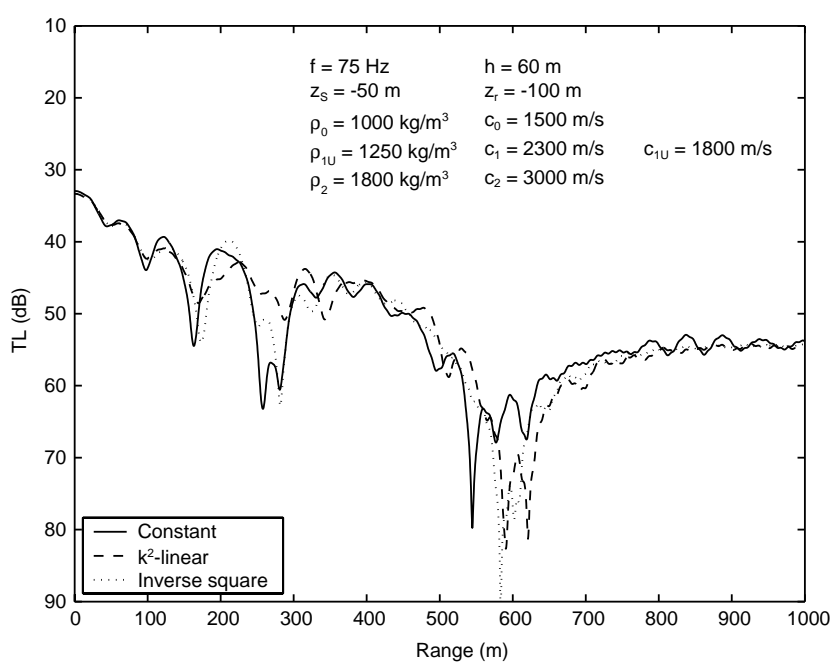

Fig. 5. TL for various sound speed distributions for frequency $75 \mathrm{~Hz}$.

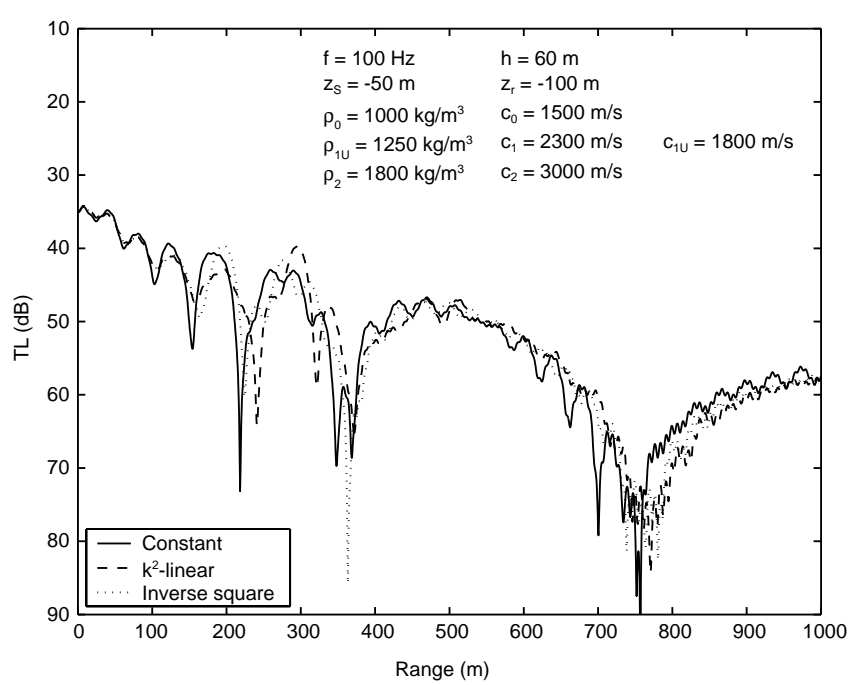

Fig. 6. TL for various sound speed distributions for frequency $100 \mathrm{~Hz}$. results for frequency $75 \mathrm{~Hz}$, and for three sediment layer thicknesses: 30,60 , and $100 \mathrm{~m}$. Again, the variation is similar to that of Figure 4).

Figure 9 demonstrates a series of results for various sediment thicknesses when the shear property of the seafloor basement is accounted for. In this case, the TL becomes higher when the sediment thickness is smaller, owing to the fact that energy may lose into the bottom with an extra mechanism of shear wave propagation. For large sediment thickness, the shear property is ineffective because the waves do not penetrate into the bottom.

Finally, Figures 10 to 12 show the complete sound

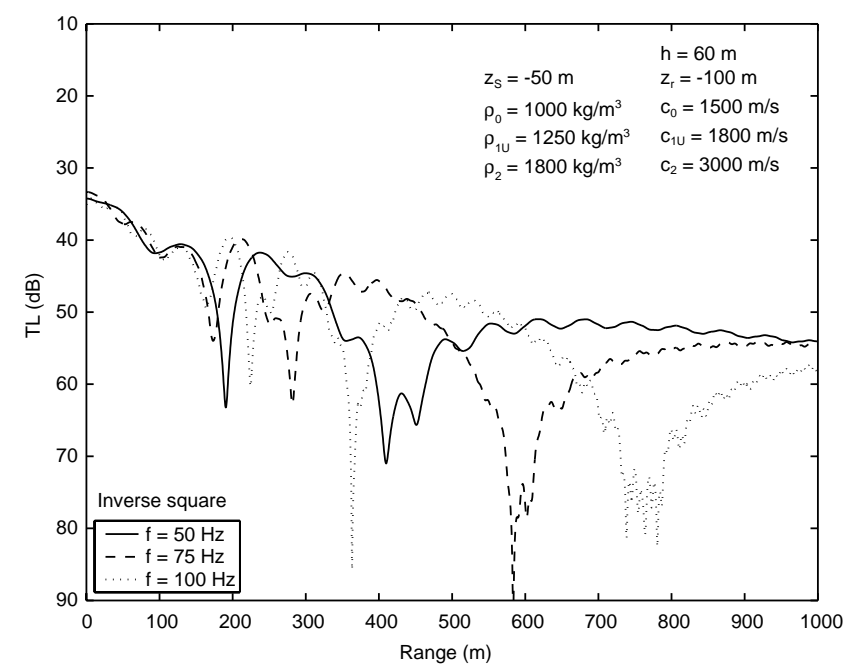

Fig. 7. TL for various frequencies for the case of inverse-square sound speed profile.

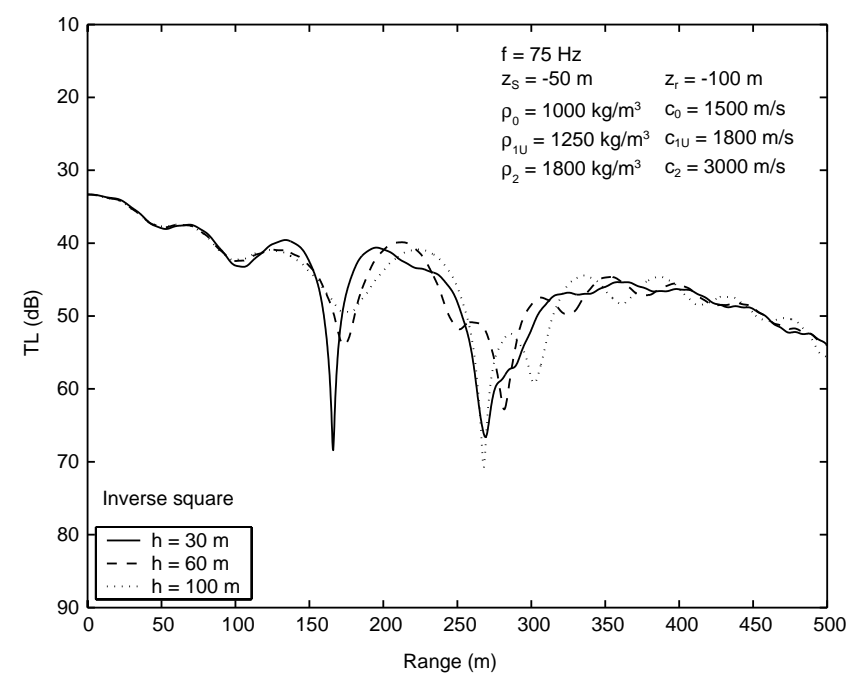

Fig. 8. TL for frequency $75 \mathrm{~Hz}$ and for three sediment layer thicknesses: 30, 60, and $100 \mathrm{~m}$. 
fields for frequency 50,75, and $100 \mathrm{~Hz}$, respectively. The sound fields in the upper and lower semi-infinite half spaces, and in the sediment layer demonstrate three different kinds of propagation pattern. In the upper medium, the interference between the source field and the reflected field results in a beam pattern whose number increases as frequency increases, while in the lower medium, the sound field is mainly penetrated outgoing waves whose energy decays quickly. In the sediment layer, the sound field demonstrates a waveguide propagation pattern, in which the energy propagation is mainly due to normal modes.

\section{CONCLUDING REMARKS}

This analysis considers the problem of acoustic point-source reflection from a flat seabed with a nonuniform fluid-like sediment layer within which the density follows a generalized-exponential variation with
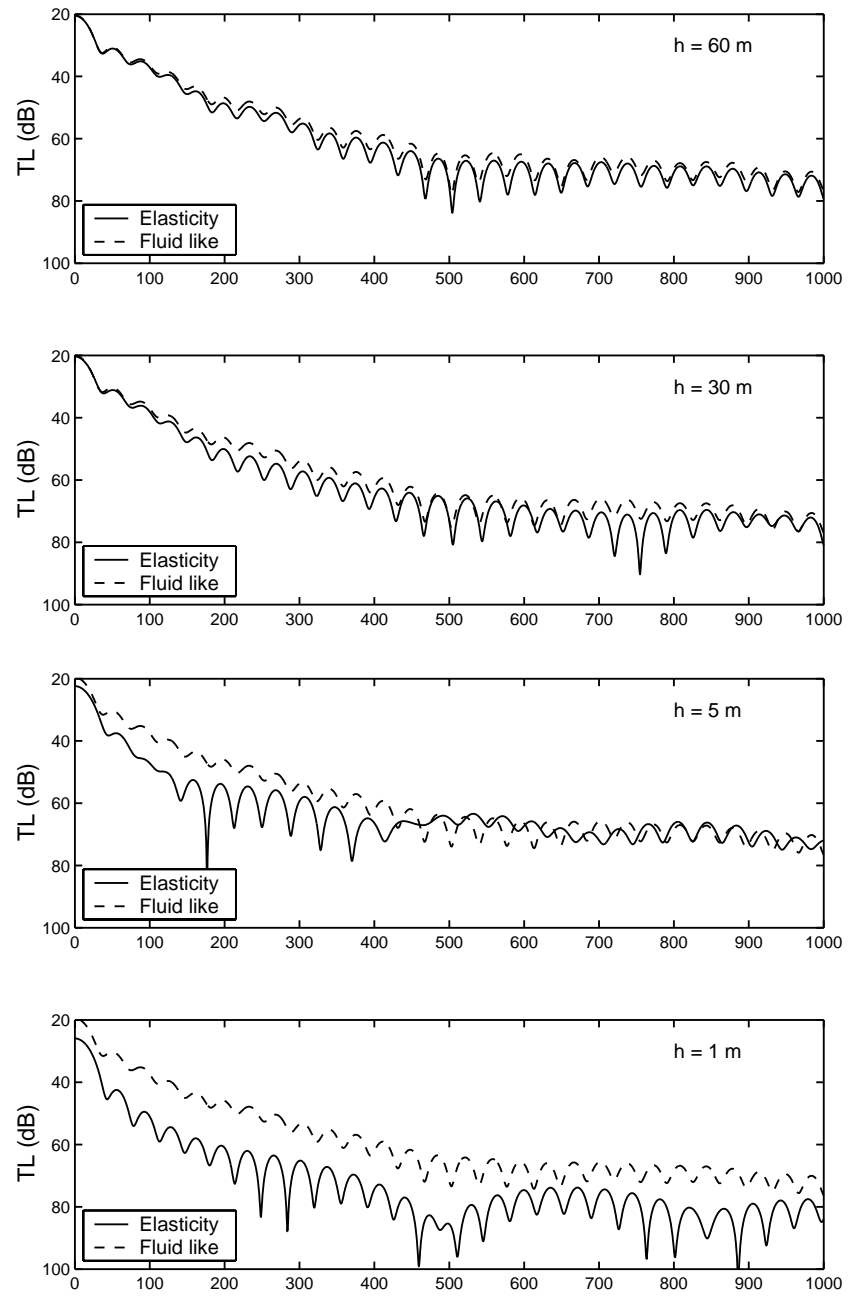

Fig. 9. Effects of basement shear property on TL for frequency $75 \mathrm{~Hz}$ and for various sediment layer thicknesses.

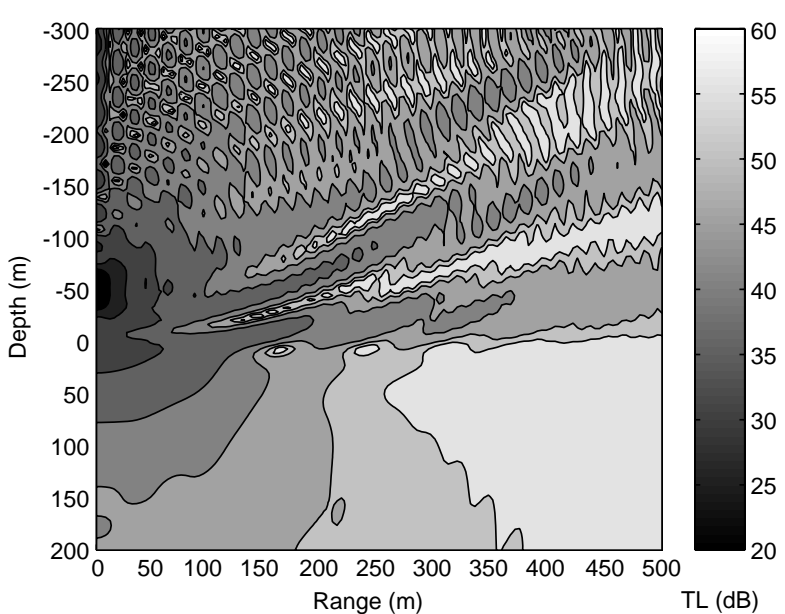

Fig. 10. Complete sound field for frequency $50 \mathrm{~Hz}$.

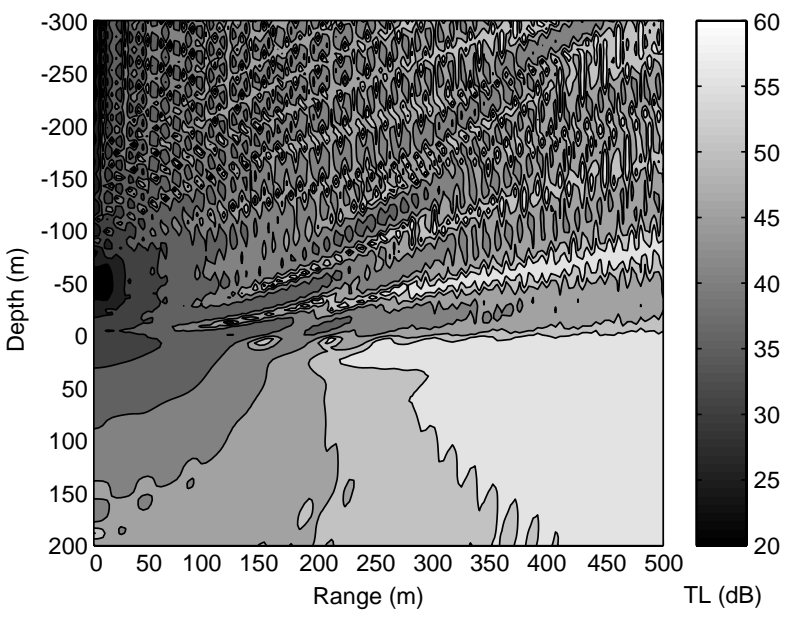

Fig. 11. Complete sound field for frequency $75 \mathrm{~Hz}$.

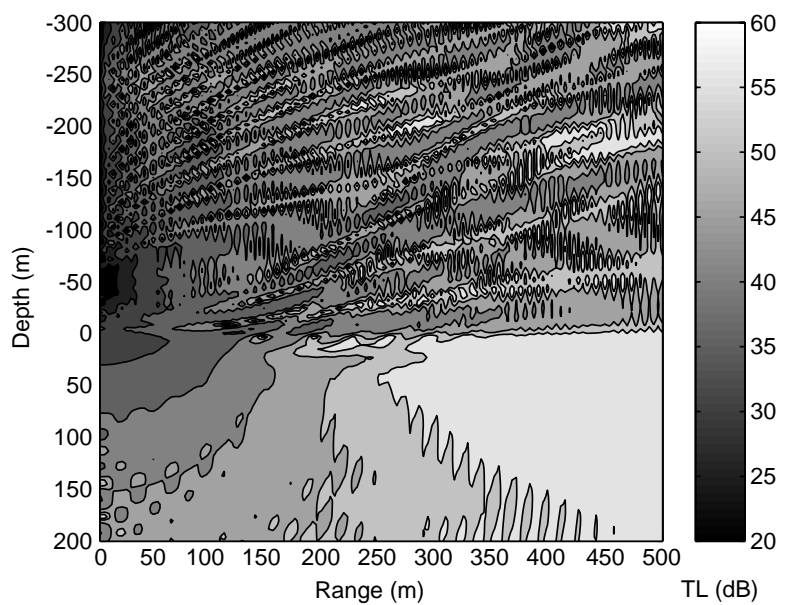

Fig. 12. Complete sound field for frequency $100 \mathrm{~Hz}$. 
depth, and the sound speed profile is either constant or varying as $k^{2}$-linear or inverse-square function. The combination of these density and sound speed profiles not only are realistic in an oceanic environment but also allow the acoustic wave equation to be solved analytically, thus facilitating the analysis of the problem. The study has shown that the different sound speed stratification in the sediment layer affects the reflection field mainly in the intermediate range of the sound field. Near or far away from the source, the reflection field is dominated by waves with propagation mechanisms that are little interactive with the sediment layer. This sheds light on the experimental procedure that a measurement should be carried out in the intermediate range, if an inversion problem for sediment stratification is in order.

\section{ACKNOWLEDGEMENTS}

This work was supported in part by National Science Council of Taiwan, R.O.C. through contract NSC91-2611-U110-005 and the Joint Project of National Cheng-Kung University and National Sun Yatsen University sponsored by the Ministry of Education of Taiwan, R.O.C. The authors would like to express our profound thanks for their financial supports.

\section{REFERENCES}

1. Boyce, W.E. and DiPrima, R.C., Elementary Differential Equations and Boundary Value Problems, John Wiley and Sons, Inc., New York (1997).

2. Brekhovskikh, L.M., Waves in Layered Media, Academic, New York (1980).

3. Hamilton, E.L., "Geoacoustic Modeling of the Sea Floor," J. Acoust. Soc. Am., Vol. 68, pp. 1313-1340 (1980).

4. Jensen, F.B., Kuperman, W.A., Porter, M.B., and
Schmidt, H., Computational Ocean Acoustics, SpringerVerlag, New York (2000).

5. Liu, J.-Y. and Huang, C.-F., "Acoustic Plane-Wave Scattering from A Rough Interface Over An Inhomogeneous Transition Fluid Layer," Ocean Eng., Vol. 28, No. 6, pp. 603-619 (2001).

6. Liu, J.-Y., Wang, C.-C., Tsai, S.-H., and Chu, C.-R., "Acoustic Wave Reflection from A Rough Seabed with a Continuously Varying Sediment Layer Overlying an Elastic Basement," J. Sound Vib. (in press)

7. Robins, A.J., "Reflection of Plane Acoustic Wave from a Layer of Varying Density," J. Acoust. Soc. Am., Vol. 87, No. 4, pp. 1546-1552 (1990).

8. Robins, A.J., "Reflection of a Plane Wave from A Fluid Layer with Continuously Varying Density and Sound Speed," J. Acoust. Soc. Am., Vol. 89, No. 4, pp. 16861696 (1991).

9. Robins, A.J., "Exact Solutions of the Helmholtz Equation for Plane Wave Propagation in A Medium with Variable Density and Sound Speed," J. Acoust. Soc. Am., Vol. 93, No. 3, pp. 1347-1352 (1993).

10. Robins, A.J., "Generation of Shear and Compression Waves in An Inhomogeneous Elastic Medium," $J$. Acoust. Soc. Am., Vol. 96, No. 3, pp. 1669-1676 (1994).

11. Tolstoy, I., "The Theory of Waves in Stratified Fluids Including the Effects of Gravity and Rotation," Rev. Mod. Phys., Vol. 35, pp. 207-230 (1963).

12. Tolstoy, I., "Effects of Density Stratification on Sound Waves," J. Geophys. Res., Vol. 70, No. 24, pp. 60096015 (1965).

13. Williams, A.O. and MacAyeal, D.R., "Acoustic Reflection from A Sea Bottom with Linearly Increasing Sound Speed," J. Acoust. Soc. Am., Vol. 66, pp. 1836-1841 (1979).

14. Wolfram, S., The Mathematica Book, Cambridge University Press, United Kingdow, United Kingdow (1996). 